Paper

\title{
Searching characteristics of chaotic neurodynamics for combinatorial optimization
}

\author{
Takafumi Matsuura ${ }^{1,2 a)}$, Kazumiti Numata ${ }^{1 b)}$, \\ and Tohru Ikeguchi ${ }^{2,3 c)}$ \\ 1 Faculty of Engineering, Tokyo University of Science \\ 1-3 Kagurazaka, Shinjyuku-ku, Tokyo 162-8601, Japan \\ 2 Graduate School of Science and Engineering, Saitama University \\ 255 Shimo-Ohkubo, Sakura-ku, Saitama-city, Saitama 338-8570, Japan \\ 3 Brain Science Institute, Saitama University \\ 255 Shimo-Ohkubo, Sakura-ku, Saitama-city, Saitama 338-8570, Japan \\ a) matsuura@ms.kagu.tus.ac.jp \\ b) numata@ms.kagu.tus.ac.jp \\ c) tohru@mail.saitama-u.ac.jp
}

Received March 30, 2012; Revised May 31, 2012; Published October 1, 2012

\begin{abstract}
An effective algorithm for solving combinatorial optimization problems by using chaotic neurodynamics has already been proposed. Although numerical simulations show that the algorithm is highly efficient, the reason behind its effectiveness has not yet been clarified. In this study, we investigated the searching characteristics of this algorithm for solving combinatorial optimization problems by employing the method of surrogate data, which is frequently used in the field of nonlinear time series analysis. We evaluated how solving abilities depend on bifurcation parameters related to the refractory effects in the chaotic neural networks. Then, we found that the considerable searching ability is decided by refractory effects after neuron firing.
\end{abstract}

Key Words: chaotic neural network, chaotic neurodynamics, refractory effect, surrogate analysis, combinatorial optimization problem

\section{Introduction}

One of the important issues in science and engineering pertains to finding solutions to combinatorial optimization problems. Although an enumeration method offers an exact solution to such problems, the number of possible combinations increases exponentially as the size of the problem increases. A typical example of such a problem is the traveling salesman problem (TSP). It has been proved that the TSP generally belongs to a class such as NP-hard. Further, it is believed that it is almost impossible to obtain an optimal solution to such a problem in a reasonable time frame. Hence, the 
development of effective approximation algorithms for solving such hard problems is necessary, even if the obtained solutions are not guaranteed to be optimal.

To find approximate solutions for combinatorial optimization problems, many heuristic algorithms have already been proposed. An effective algorithm that introduces chaotic neurodynamics for exploring solution spaces has also been proposed [1-7]. The algorithm is primarily based on the concept that the execution of a heuristic algorithm or a local search algorithm, such as the 2-opt algorithm for solving TSP, is controlled by chaotic neurodynamics. Further, it has already been shown that if the concept is introduced, good near-optimal solutions can be found, because the chaotic dynamics effectively avoids undesirable traps of the local search algorithm into local minima. Then, the local search algorithm controlled by the chaotic dynamics is very effective not only for TSP $[1,2]$ but also for other NP-hard problems such as the quadratic assignment problem (QAP) [3], the vehicle routing problem (VRP) $[4,5]$, and the motif extraction problem (MEP) [6, 7].

In this algorithm [1-7], a chaotic neural network model is used for realizing a chaotic search; the model qualitatively realizes an important biological feature of a real neuron - the refractory effect [8]. Because of this effect, a neuron hardly fires again for a certain period of time after it has fired or emitted a spike. In the chaotic searches applied to the TSP [1,2], QAP [3], VRP [4,5], and MEP [6, 7], the exploration of the solution space of the problem is controlled by the firing of the chaotic neuron. If the neuron fires, the state of the searching space changes. Subsequently, the selection of the same solution can be avoided after the firing of the corresponding neuron due to the refractory effect.

Thus, the refractory effect has a memory effect similar to that in the case of the tabu search, which is one of the strongest metaheuristic algorithms $[9,10]$. Hence, it can be concluded that the refractory effect plays a significant role in finding solutions for the above-mentioned problems. In other words, the refractory effect is an important factor in solving combinatorial optimization problems because it forces a change in the searching state, which leads to an effective escape from a local minimum.

Although it has already been shown that the chaotic search is very effective in finding good approximate or near-optimal solutions for combinatorial optimization problems such as TSP, QAP, VRP and MEP, the characteristics required for the algorithm to be highly efficient have not been confirmed yet. If these characteristics are identified, the algorithm can be improved further. From these points of view, analyses on the chaotic dynamics in chaotic search have been studied recently $[11,12]$. In Refs. $[11,12]$, internal states of chaotic neuron have been analyzed. As a result, it was clarified that chaotic search method obtains better solution, when internal states of the chaotic neuron behave as the "edge of chaos."

However, it is not enough to analyze only the internal state of neuron, because the internal state of the chaotic neuron is composed of two parts; a gain effect and a refractory effect. The refractory effect realizes an efficiency of the chaotic search methods, because the refractory effect works to escape from local minima and controls to move the solution space. Analysis on the refractory effect could lead to deeper understanding of why the chaotic dynamics show high performance for combinatorial optimization than analysis on internal state. To analyze chaotic dynamics, several statistical indices are often used such as Lyapunov exponent, attractor dimension, and so on. However, these indices are not suitable in our case, because they are usually applied to state variables of dynamical systems: namely, internal states of the chaotic neural network. Because the internal states are decomposed into two parts, the gain effect and the refractory effect, it is difficult to directly analyze the internals states by using the conventional measures. Then, it needs to analyze its dynamics by a different analysis method.

Thus, in this paper, we analyze the refractory effect of the chaotic neuron to solve the issue. We investigated which characteristics are essential for high performance of the chaotic search by introducing a technique from the field of nonlinear time series analysis - the method of surrogate data [13]. We used the method of surrogate data as a statistical control in the above-mentioned investigation. We adopted the following strategy: the time series that represented the refractory effect of a chaotic neuron was replaced with a surrogate time series of the refractory effect, for example, by a random shuffling or by a controlled shuffling of the original refractory time series. Subsequently, we compared the performance of the original refractory effect with that of the surrogate refractory effect. 
In the chaotic search, two bifurcation parameters control the refractory effect. The first one decides the strength of the refractory effect after neuron firing. The second one controls the decay speed of the refractory effect. Although we have already reported preliminary results in case of solving MEP [14] by investigating only one parameter, we have also found that the performance of the chaotic search changes considerably, even if the values of each parameter are changed slightly. Hence, in this paper, we systematically investigated the refractory effect by changing these bifurcation parameters. As a result, we show that the strength of refractory effect after neuron firings is an important factor to realize an efficient exploration of the searching space.

\section{Motif extraction problem}

In April 2003, the human genome project was completed. This project generated large data sets of genomic sequences. The human genome consists of approximately three billion base pairs and approximately 25,000 genes. One of the primary issues in the field of bioinformatics is the identification of important parts in which biological information is embedded. In general, it is considered that biologically essential alignments appear repeatedly within a biological sequence, for example, DNA, RNA, and protein sequences. Therefore, one of the popular analyses involves finding a common and conserved pattern. Such a pattern is often called a motif.

To find the motif, multiple alignment problem is solved. Although the multiple alignment problem is divided into two types; global multiple alignment and local multiple alignment, we focus on the local multiple alignment in this paper. The definition of the local multiple alignment can be mathematically described as follows: We have a biological data set $S=\left\{s_{1}, s_{2}, \ldots, s_{N}\right\}$, where $s_{i}$ is the $i$ th sequence (Fig. 1). Each sequence consists of $m_{i}(i=1,2, \ldots, N)$ elements. In the case of DNA or RNA, the elements correspond to four bases; while in the case of protein sequences, they correspond to 20 amino acids. The aim of the local multiple alignment is to find a set of motifs $V=\left\{v_{1}, v_{2}, \cdots, v_{N}\right\}$, where the length of the motif is $L$. In this paper, we assume that only one motif exists in all sequences and no gaps are included in the motifs (Fig. 1) [15]. Of course, alignment of the motifs and length of the motifs are unknown. The set of motifs is decided by maximizing the following objective function:

$$
E=\frac{1}{L} \sum_{k=1}^{L} \sum_{\omega \in \Omega} f_{k}(\omega) \log _{2} \frac{f_{k}(\omega)}{p(\omega)},
$$

where $f_{k}(\omega)$ is the appearance frequency of an element $\omega \in \Omega$ at the $k$ th column in a set $V$; $\Omega$ is a set of bases or a set of amino acids; $p(\omega)$ is the background probability of the element $\omega$ in the set $S$ and is calculated by dividing the number of an element $\omega$ in the set $S$ by the number of all elements $\sum_{i=1}^{N} m_{i}$. In this paper, we define $0 \log _{2} 0 \equiv 0$.

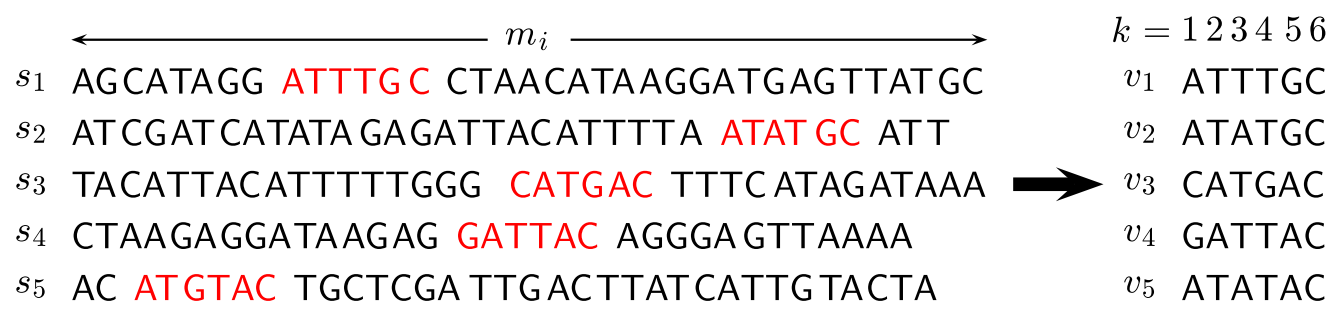
$\leftarrow L \rightarrow$

Fig. 1. Example of the motif extraction problem. This example includes $N=5$ sequences $\left(s_{1}, s_{2}, \ldots, s_{5}\right)$ and each sequence is composed of $m_{i}$ bases: A, $\mathrm{C}, \mathrm{G}$, and $\mathrm{T}$ denote adenine, cytosine, guanine, and thymine, respectively. The length of motif is $L=6$. In this example, a set of motifs $V=\left\{v_{1}, v_{2}, \ldots, v_{5}\right\}$ is extracted from the sequences $s_{1}, s_{2}, \ldots, s_{5}$. At the first column $(k=1)$ in the set $V$, the number of the base $\mathrm{A}$ is 3 . Then, $f_{1}(\mathrm{~A})=\frac{3}{5}=0.6$. Other values are $f_{1}(\mathrm{C})=0.2, f_{1}(\mathrm{G})=0.2, f_{2}(\mathrm{~A})=0.4, f_{2}(\mathrm{~T})=0.6, f_{3}(\mathrm{~A})=0.4, f_{3}(\mathrm{~T})=0.6$, $f_{4}(\mathrm{G})=0.2, f_{4}(\mathrm{~T})=0.8, f_{5}(\mathrm{~A})=0.6, f_{5}(\mathrm{G})=0.4$, and $f_{6}(\mathrm{C})=1.0$. The other cases are 0. 


\subsection{Chaotic motif sampler}

Several approximation methods have been developed for the MEP. Gibbs sampling is one of the most popular heuristic algorithms and is now used extensively in the field of bioinformatics [17]. This algorithm uses a stochastic search for exploring possible states.

Although Gibbs sampling often works very well, we have developed a different heuristic search for the MEP - Chaotic Motif Sampler (CMS) [6,7]. The CMS introduces chaotic neurodynamics in deciding a new motif position. To realize the chaotic search, we first develop a chaotic neural network [8] composed of $\sum_{i=1}^{N}\left(m_{i}-L+1\right)$ neurons (Fig. 2).

In the CMS, the firing of a chaotic neuron encodes the movement of the head position of a motif candidate to the corresponding position (Fig. 2). The $(i, j)$ th chaotic neuron is defined by

$$
\begin{aligned}
x_{i j}(t) & =f\left(y_{i j}(t)\right), \\
f(y) & =\frac{1}{1+e^{-\frac{y}{\epsilon}}}, \text { and } \\
y_{i j}(t) & =\xi_{i j}(t)+\zeta_{i j}(t),
\end{aligned}
$$

where $x_{i j}(t)$ is the output of the $(i, j)$ th chaotic neuron and $y_{i j}(t)$ is the internal state of the $(i, j)$ th neuron at time $t$. If $x_{i j}(t)>\frac{1}{2}$, the $(i, j)$ th chaotic neuron is firing. The internal state of the chaotic neuron [8] is decomposed into two parts- $\xi_{i j}(t)$ and $\zeta_{i j}(t)$ - each of which represents the different effects determining the firing of the neuron in the algorithm: the gain effect and the refractory effect, respectively.

The first part, $\xi_{i j}(t)$, which expresses the gain effect, is defined as

$$
\begin{aligned}
\xi_{i j}(t+1) & =\beta\left(E_{i j}(t)-\hat{E}\right), \text { and } \\
E_{i j}(t) & =\frac{1}{L} \sum_{k=1}^{L} \sum_{\omega \in \Omega} f_{k}(\omega) \log _{2} \frac{f_{k}(\omega)}{p(\omega)},
\end{aligned}
$$

where $\beta$ is the scaling parameter of the effect; $E_{i j}(t)$, an objective function or the relative entropy score when a candidate motif position is moved to the $j$ th position in the sequence $s_{i} ; \hat{E}$, the entropy score of the current state; $f_{k}(\omega)$, the number of appearances of an element (a base in the case of DNA or RNA and an amino acid in the case of a protein) $\omega \in \Omega$ the at the $k$ th position of the subsequences; $p(\omega)$, the background probability of appearance of the element $\omega$; and $\Omega$, a set of elements $\left(\Omega_{\mathrm{BASE}}=\right.$ $\{\mathrm{A}, \mathrm{C}, \mathrm{G}, \mathrm{T}\})$ or a set of amino acids $\left(\Omega_{\mathrm{ACID}}=\{\mathrm{N}, \mathrm{S}, \mathrm{D}, \mathrm{Q}, \mathrm{E}, \mathrm{T}, \mathrm{R}, \mathrm{H}, \mathrm{G}, \mathrm{K}, \mathrm{Y}, \mathrm{W}, \mathrm{C}, \mathrm{M}, \mathrm{P}, \mathrm{F}, \mathrm{A}, \mathrm{V}, \mathrm{L}, \mathrm{I}\}\right)$. The quantity on the righthand side of Eq. (5) becomes positive if a new motif candidate position is better than the current state. Thus, the chaotic neuron is encouraged to fire, if the first part, $\xi_{i j}(t)$, is used.

The second part, $\zeta_{i j}(t)$, qualitatively realizes the refractoriness of the neuron. Refractoriness is one of the important properties of real biological neurons; once a neuron fires, it becomes difficult for the neuron to fire again for a certain period of time. The second part is expressed as follows:

$$
\begin{aligned}
\zeta_{i j}(t+1) & =-\alpha \sum_{d=0}^{t} k_{r}^{d} x_{i j}(t-d)+\theta \\
& =-\alpha x_{i j}(t)+k_{r} \zeta_{i j}(t)+\theta\left(1-k_{r}\right)
\end{aligned}
$$

where $\alpha$ is a positive parameter; $k_{r}$, a decay parameter that assumes values between 0 and 1 ; and $\theta$, a threshold value. Hence, in Eq. (7), $\zeta_{i j}(t+1)$ expresses the refractory effect with a factor $k_{r}$ because the more the neuron has fired in its past, the more negative the first term of the righthand side of Eq. (7) becomes, which in turn depresses the value of $\zeta_{i j}(t+1)$ and leads the neuron to a relatively resting state.

On the basis of the above-mentioned internal states, we construct an algorithm for extracting motifs in the following manner: Let us consider a set of $N$ sequences of lengths $m_{i}(i=1,2, \ldots, N)$; further, let the length of a subsequence (motif) be $L$ (Fig. 2). 
1. The position of an initial subsequence $v_{i j}\left(i=1,2, \ldots, N ; j=1,2, \ldots, m_{i}-L+1\right)$ is randomly selected from the $i$ th sequence.

2. The $i$ th sequence $s_{i}$ is selected cyclically.

3. For a selected sequence $s_{i}$ at the second step, the position of the motif candidate is changed to a new position. Then, $y_{i j}(t+1)$ is calculated from the first neuron $(j=1)$ to the last neuron $\left(j=m_{i}-L+1\right)$ in the sequence $s_{i}$. A neuron whose internal state is maximum is selected. If the selected neuron fires $\left(x_{i j}(t+1)>\frac{1}{2}\right)$, corresponding candidate position becomes a new motif position and the value of $\hat{E}$ is updated. But if the selected neuron does not fire, the motif position does not change.

4. Steps 2 and 3 are repeated for a sufficient number of iterations. Then, the CMS outputs the obtained best solutions (motifs).

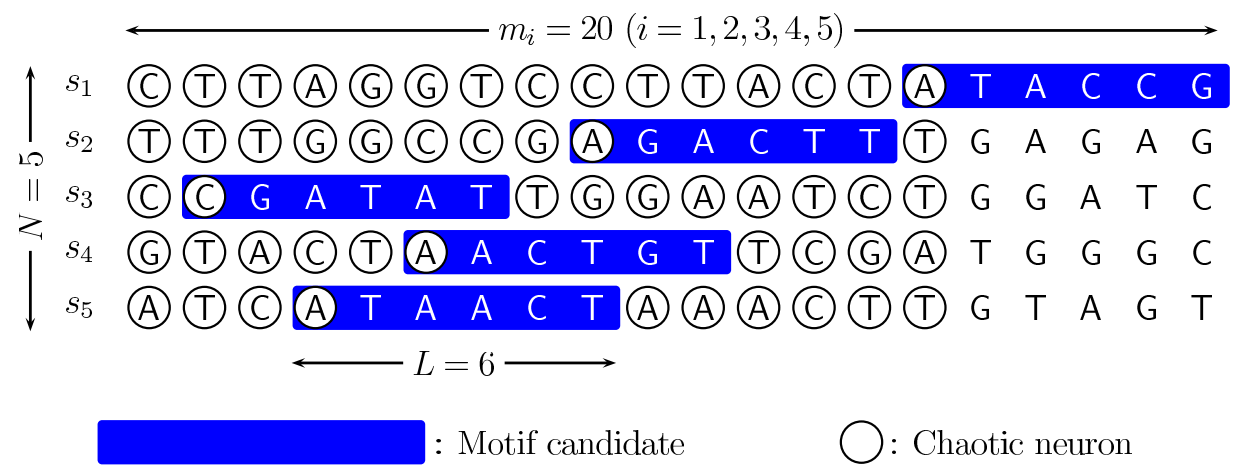

Fig. 2. Coding scheme of motif positions in the chaotic motif sampler. A chaotic neuron is assigned to a head position of all motif candidates. In this example, the number of DNA sequences is $N=5$, each sequence is composed of $m_{i}=20(i=1,2, . ., N)$ bases, and a motif length is $L=6$. Then, the chaotic motif sampler is constructed by $\sum_{i=1}^{N}\left(m_{i}-L+1\right)=\sum_{i=1}^{5}(20-6+1)=75$ chaotic neurons.

\section{Results}

To investigate the searching properties of the CMS and determine the refractory effects under real-life situation, we use real protein sequences $[17,18]$. This data set includes 30 sequences $(N=30)$, and the $i$ th sequence is composed of $m_{i}(i=1,2, \ldots, N)$ amino acids. In the data set of 30 sequences, the correct location of the motif has already been identified $[17,18]$. The length of the motif is 18 $(L=18)$. If the motifs are extracted exactly, the relative entropy score (Eq. (6)) will assume the value of 1.65 in this case.

In the CMS, the parameter $\alpha$ controls the strength of the refractory effect after neuron firing, the parameter $k_{r}$ is the decay parameter of the refractory effect, and the parameter $\theta$ is threshold of the refractory effect. Therefore, we extract the motifs from the data set by using the CMS with several values of $\alpha, k_{r}$, and $\theta$. The other parameters in the CMS are set to $\beta=30.0$ and $\epsilon=0.01$.

\subsection{Results obtained by using CMS}

Figure 4 shows the result of exploring the parameter space for finding motifs by using the CMS. The CMS can find the motifs with a high success rate for many parameter sets. These results indicate that the CMS can extract motifs effectively with a good performance if the parameters $\alpha, k_{r}$, and $\theta$ are set appropriately. Figure 3 shows Lyapunov exponents of a single chaotic neuron in CMS, namely the case that $\xi_{i j}(t)=0$. From Fig. 3, its dynamics can be chaotic depending on the values of parameters $\alpha$ and $k_{r}$. However, from Figs. 3 and 4 , even if the Lyapunov exponents are positive, the CMS cannot 
find motifs. It indicates that the solving performance of the CMS does not depend on the values of Lyapunov exponents due to external input $\xi_{i j}(t)$.
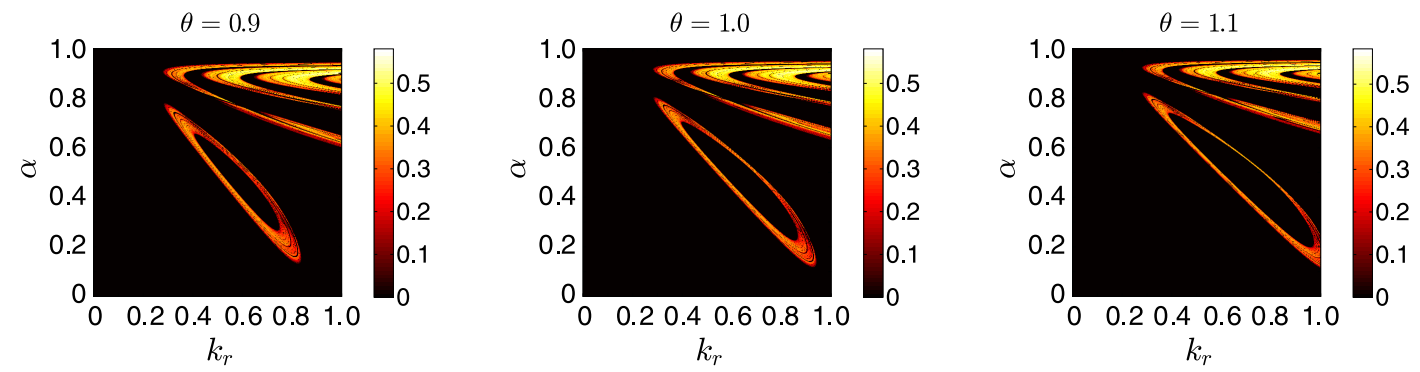

Fig. 3. Lyapunov exponents of a chaotic neuron $(\epsilon=0.02)$. Negative values are colored in black and positive values are coded by multi colors shown in the color bars.
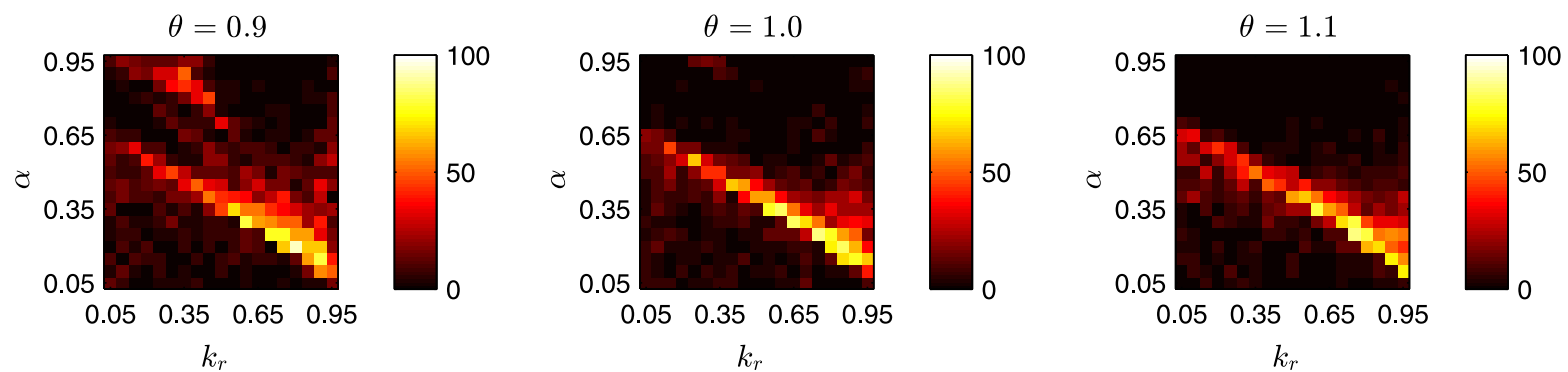

Fig. 4. Performance of the chaotic motif sampler. The average probabilities (\%) of finding motifs in 50 trials are indicated by shading. If the motifs are correctly found in 40 trials, the probability is $40 / 50=80 \%$.

\subsection{Analysis on the refractory effect}

\subsubsection{Surrogate analysis of time series $\zeta_{i j}(t)$}

One of the possible reasons for the high success rates of the CMS is that the refractory effect efficiently controls the neuron firing. Figure 5 shows an example time series $\zeta_{i j}(t)$ of the refractory effect of the CMS.

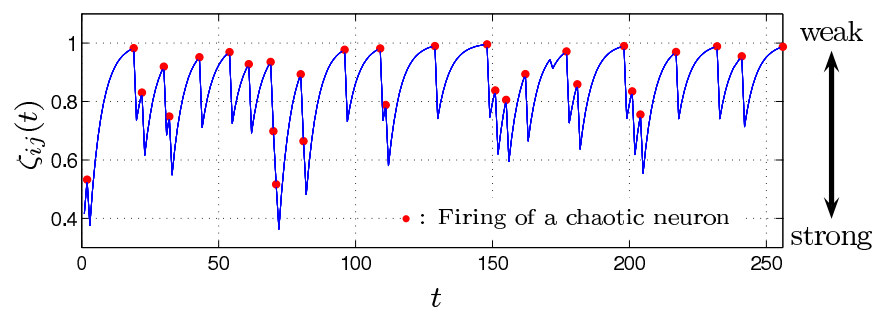

Fig. 5. Example time series of refractory effect $\zeta_{i j}(t)$ in a chaotic neuron. Red dots on the time series represent the times at which the $(i, j)$ th neuron fires. The refractory effect increases suddenly after the neuron firings and it then decreases gradually with an exponential decay.

In Fig. 5, it is easily observed that the refractory effect increases suddenly after neuron firings and it then decreases gradually with an exponential decay. Figure 6 shows the auto-correlation of $\zeta_{i j}(t)$ of the chaotic neuron in the CMS. It has already been reported that long-time correlation of the dynamics is important for the efficiency of the chaotic search. However, we cannot observed such phenomenon in the CMS (Fig. 6). This result indicates that the correlation function is not important from the view point of finding motifs efficiently.

To investigate the refractory effects of chaotic neurons, we replace the original refractory effect time series $\zeta_{i j}(t)$ in Eq. (4) with surrogate refractory effects that have the same first- or second-order 


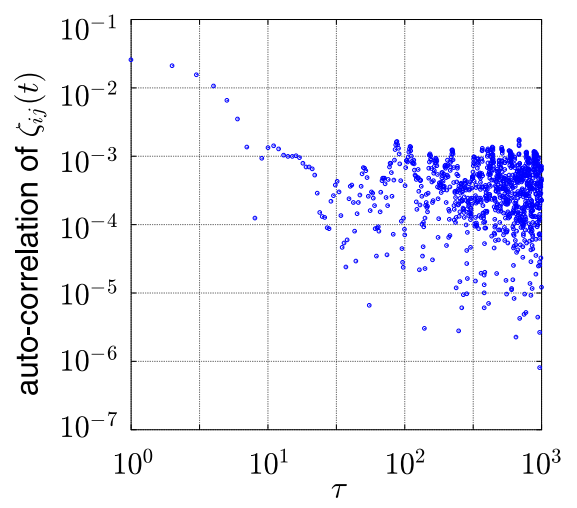

Fig. 6. Example auto-correlation of time series of refractory effect $\zeta_{i j}(t)$ in a chaotic neuron. $\tau$ is the time lag.

statistics as the original refractory effect. Further, to produce the surrogate refractory effects, we introduce the method of surrogate data [13], which is often used in the field of nonlinear time series analysis. The five surrogates are described as follows:

RS: Random Shuffle

$y_{i j}(t)=\xi_{i j}(t)+\zeta_{i j}^{\prime}(t)$, where $\zeta_{i j}^{\prime}(t)$ is a randomly shuffled time series of $\zeta_{i j}(t)$ (Fig. 7(A)). RS has only the same first-order statistics as the original refractory effect time series $\zeta_{i j}(t)$ (Fig. $7(\mathrm{~B})$ and $(\mathrm{C})$ ).

FT: Fourier Transform

$y_{i j}(t)=\xi_{i j}(t)+\hat{\zeta}_{i j}^{\prime}(t)$, where $\hat{\zeta}_{i j}^{\prime}(t)$ is a Fourier transformed surrogate [13] time series of the original refractory effect time series $\zeta_{i j}(t)$ (Fig. 7(A)). The FT surrogate has the same correlation function as that of the original $\zeta_{i j}(t)$, but it destroys the frequency histogram of the original $\zeta_{i j}(t)($ Fig. $7(\mathrm{~B})$ and $(\mathrm{C}))$.

FS: Fourier Shuffle

$y_{i j}(t)=\xi_{i j}(t)+\tilde{\zeta}_{i j}^{\prime}(t)$, where $\tilde{\zeta}_{i j}^{\prime}(t)$ is a Fourier shuffled surrogate [13] of the original refractory effect time series $\zeta_{i j}(t)$ (Fig. 7(A)). The FS surrogate produces a time series that preserves both first- and second-order statistics of $\zeta_{i j}(t)$ (Fig. 7(B) and (C)).

IAAFT: Iterated Amplitude Adjusted Fourier Transform

$y_{i j}(t)=\xi_{i j}(t)+\dot{\zeta}_{i j}^{\prime}(t)$, where $\dot{\zeta}_{i j}^{\prime}(t)$ is an iterated amplitude adjusted Fourier transformed surrogate [13] of the original refractory effect $\zeta_{i j}(t)$ (Fig. 7(A)). The IAAFT surrogate has the same first-order statistics and preserves the second-order statistics of $\zeta_{i j}(t)$ (Fig. 7(B) and $(\mathrm{C})$ ). However, the preservation of the correlation structure by using the IAAFT surrogate is superior to that using the FS surrogate.

First, we extract a motif by using the RS surrogate that has the same frequency histogram as that of the original refractory effect $\zeta_{i j}(t)$. To preserve the frequency histogram, we construct a surrogate time series by randomly shuffling all the temporal indices of the original $\zeta_{i j}(t)$. Figure $7(\mathrm{~A})$ shows an example of a randomly shuffled time series of $\zeta_{i j}(t)$; Fig. 7(B) shows its frequency histogram; and Fig. $7(\mathrm{C})$ shows its correlation function. If performance of the RS surrogate is the same as the that of CMS, an efficiency of the chaotic search is realized by the frequency histogram of the refractory effect. The performance of RS surrogate is shown in Fig. 8(A). From the results, the motifs cannot be extracted by the RS surrogate (Fig. 8(A)).

In the case of the RS surrogate, although the frequency histogram (the first-order statistics) is the same as that of the original $\zeta_{i j}(t)$, the correlation structure (the second-order statistics) is different (Fig. 7(B) and (C)). The low performance of the RS surrogate may be attributed to this difference. Then, we extract motifs by using the FT surrogate time series whose correlation function is exactly the same as that of the original $\zeta_{i j}(t)$ (Fig. 7(A), (B), and (C)). As a result, we cannot identify the motifs by using the FT surrogate time series (Fig. 8(B)). We presume that the two characteristics, 
A
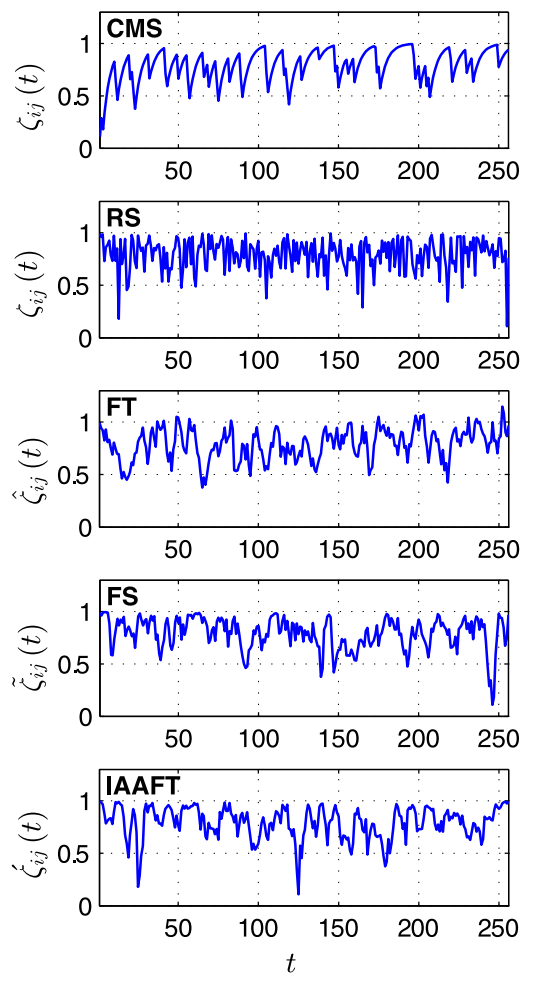

B
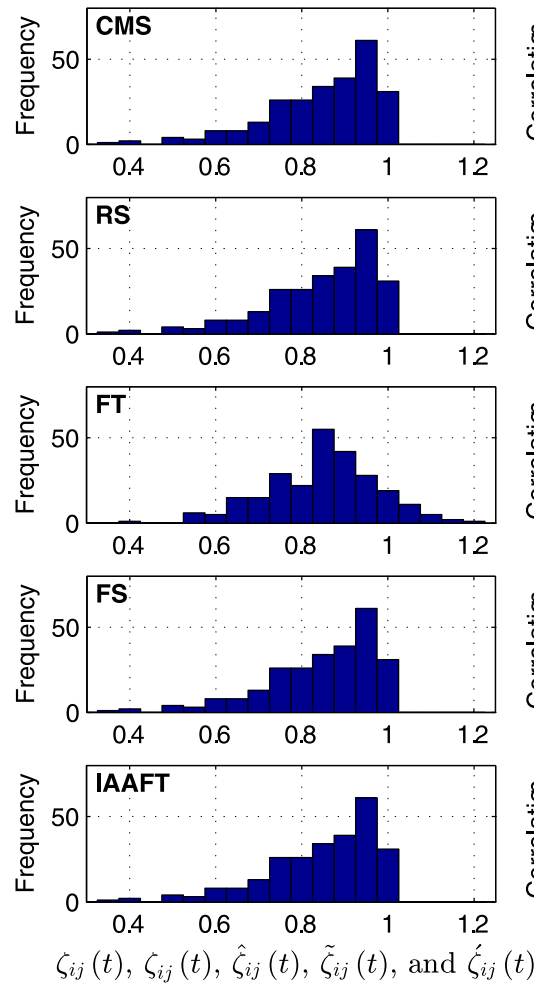

C
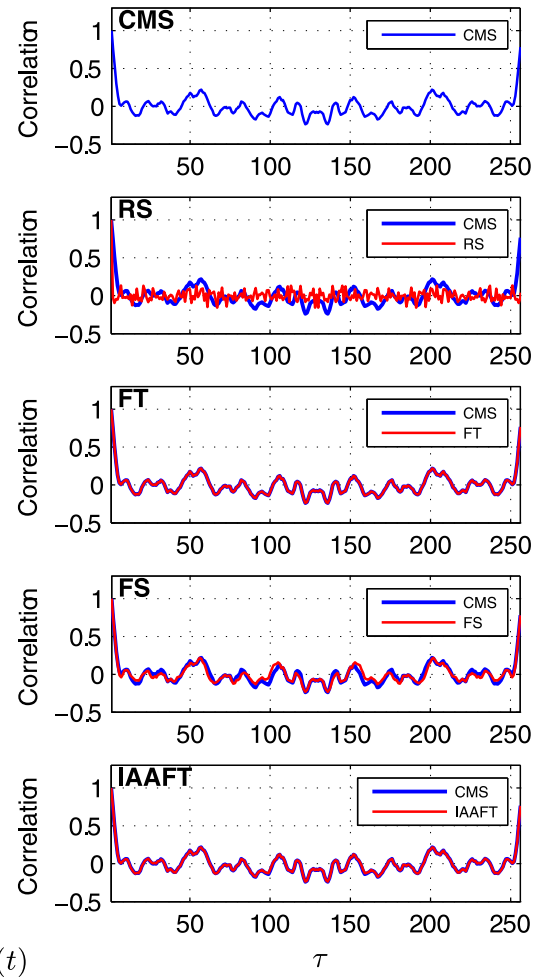

Fig. 7. Characteristics of the surrogate refractory effects. (A) time series of the original refractory effect $\zeta_{i j}(t)$ and its surrogates refractory effects: random shuffle surrogate (RS), Fourier transform surrogate (FT), Fourier shuffle surrogate (FS), and iterated amplitude adjusted Fourier transform surrogate (IAAFT). (B) Frequency histograms of the time series of the original refractory effect $\zeta_{i j}(t)$ and its surrogate refractory effects; $\zeta_{i j}^{\prime}(t), \hat{\zeta}_{i j}^{\prime}(t),{\tilde{\zeta_{i j}}}^{\prime}(t)$ and $\dot{\zeta}_{i j}{ }^{\prime}(t)$. (C) Correlation function of the original refractory effect $\zeta_{i j}(t)$ and its surrogate refractory effects. $\tau$ is the time lag.

the frequency histogram and the correlation function, are important from the view point of finding motifs efficiently.

Then, we extract the motifs by using the FS surrogate time series whose frequency histogram is perfectly the same as that of the original $\zeta_{i j}(t)$ and correlation function is almost the same as that of the original $\zeta_{i j}(t)$ (Fig. 7(A), (B), and (C)). As a result, the motifs are not extracted again (Fig. 8(C)).

Finally, for approximating the correlation structure of the original $\zeta_{i j}(t)$ more precisely, we produce the IAAFT surrogate time series (Fig. 7(A), (B), and (C)). The IAAFT surrogate can preserve the second-order statistics of the original $\zeta_{i j}(t)$ better than the FS (Fig. $7(\mathrm{C})$ ). However, the IAAFT surrogate refractory effect cannot find the motifs as well as the CMS (Fig. 8(D)).

The results presented in this section indicate that the surrogate refractory series cannot effectively control the neuron firings and the exploration in the solution space. The primary difference between the surrogate refractory effects and the original $\zeta_{i j}(t)$ is now clear. The values of the original $\zeta_{i j}(t)$ recover exponentially with time and become strong after neuron firings (Fig. 5). In contrast, the surrogate refractory effects do not exhibit such characteristic behavior because they are decided without considering the previous states of the neurons. It may be important to strengthen the refractory effects after the neuron firing.

\subsection{Surrogate analysis of the strength of the refractory effects after neuron firing}

In this section, we analyze the strength of the refractory effects after neuron firing. To investigate the refractory effects after neuron firings, at first, we make a time series $R_{i j}(n)$, which is the $n$th value of 

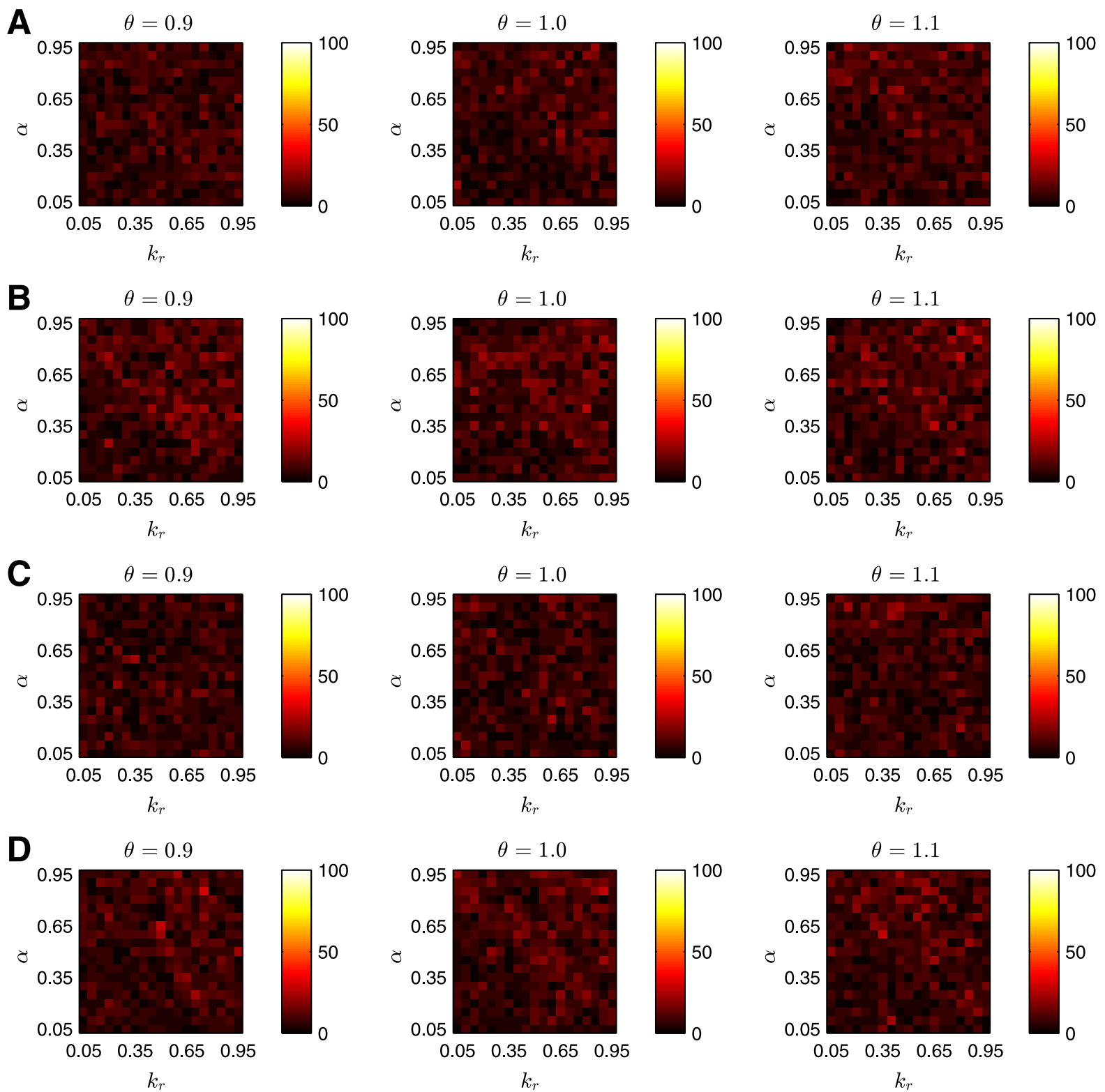

Fig. 8. Performance of the surrogate refractory effects. The average probabilities $(\%)$ of finding motifs in 50 trials are indicated by the shading. To analyze the refractory effect in the chaotic neuron, the bifurcation parameters related to the refractory effect, $\alpha, k_{r}$, and $\theta$ are set to various values. The other parameters are fixed to $\beta=30.0$ and $\epsilon=0.01$. Each surrogate refractory effect is made by original refractory effect $\zeta_{i j}(t)$. (A) performance of the random shuffle surrogate, (B) performance of the random Fourier transform surrogate, (C) performance of the random Fourier shuffle surrogate, and (D) performance of the iterated amplitude adjusted Fourier transform are shown.

the strength of the refractory effects after the $n$th firing of the $(i, j)$ th neuron in the CMS (Fig. 9). In the CMS, if the $i j$ th neuron fires at time $t\left(x_{i j}(t)>0.5\right)$, at the next time, the refractory effect of the $i j$ th neuron becomes stronger depending on the value of $x_{i j}(t)$ (Fig. 9). In Eq. (8), the refractory effect of after neuron firing corresponds to $\alpha x_{i j}(t)$, namely $R_{i j}(n)=-\alpha x_{i j}(t)$.

To investigate the refractory effects after neuron firings, we replace the first term of Eq. (8) with the following three cases:

Case A:

$$
\zeta_{i j}(t+1)= \begin{cases}k_{r} \zeta_{i j}(t)+\theta\left(1-k_{r}\right), & x_{i j}(t) \leq 0.5 \\ k_{r} \zeta_{i j}(t)+\theta\left(1-k_{r}\right)-R_{i j}^{\prime}(n), & x_{i j}(t)>0.5,\end{cases}
$$

where $R_{i j}^{\prime}(n)$ is a random shuffled time series of $R_{i j}(n)$ (Fig. 9). Here, $R_{i j}(n)$ is the $n$th value 
of the strength of the refractory effects after the $n$th firing of the $(i, j)$ th neuron in the CMS (Fig. 9). In the CMS, if $x_{i j}(t)>\frac{1}{2}$, the $i j$ th neuron fires. Then, the values of the $R_{i j}(n)$ are $\frac{\alpha}{2}<R_{i j}(n)<\alpha$.

Case B:

$$
\zeta_{i j}(t+1)= \begin{cases}k_{r} \zeta_{i j}(t)+\theta\left(1-k_{r}\right), & x_{i j}(t) \leq 0.5 \\ k_{r} \zeta_{i j}(t)+\theta\left(1-k_{r}\right)-\tilde{A}_{i j}(t), & x_{i j}(t)>0.5\end{cases}
$$

where $\tilde{A}_{i j}(t)$ is a uniform random number whose dynamic range is adjusted to that of $R_{i j}(n)$ for all neurons.

\section{Case C:}

$$
\zeta_{i j}(t+1)= \begin{cases}k_{r} \zeta_{i j}(t)+\theta\left(1-k_{r}\right), & x_{i j}(t) \leq 0.5 \\ k_{r} \zeta_{i j}(t)+\theta\left(1-k_{r}\right)-A(t), & x_{i j}(t)>0.5\end{cases}
$$

where $A(t)$ is a uniform random number whose dynamic range is between 0 and 1.

Figure 10 shows the frequency histogram of the time series of $R_{i j}(n), R_{i j}^{\prime}(n), \tilde{R}_{i j}(n)$, and $A_{i j}(n)$, when the values of parameters in CMS are set to $\alpha=0.30, k_{r}=0.90, \theta=1.0, \beta=30$, and $\epsilon=0.01$.

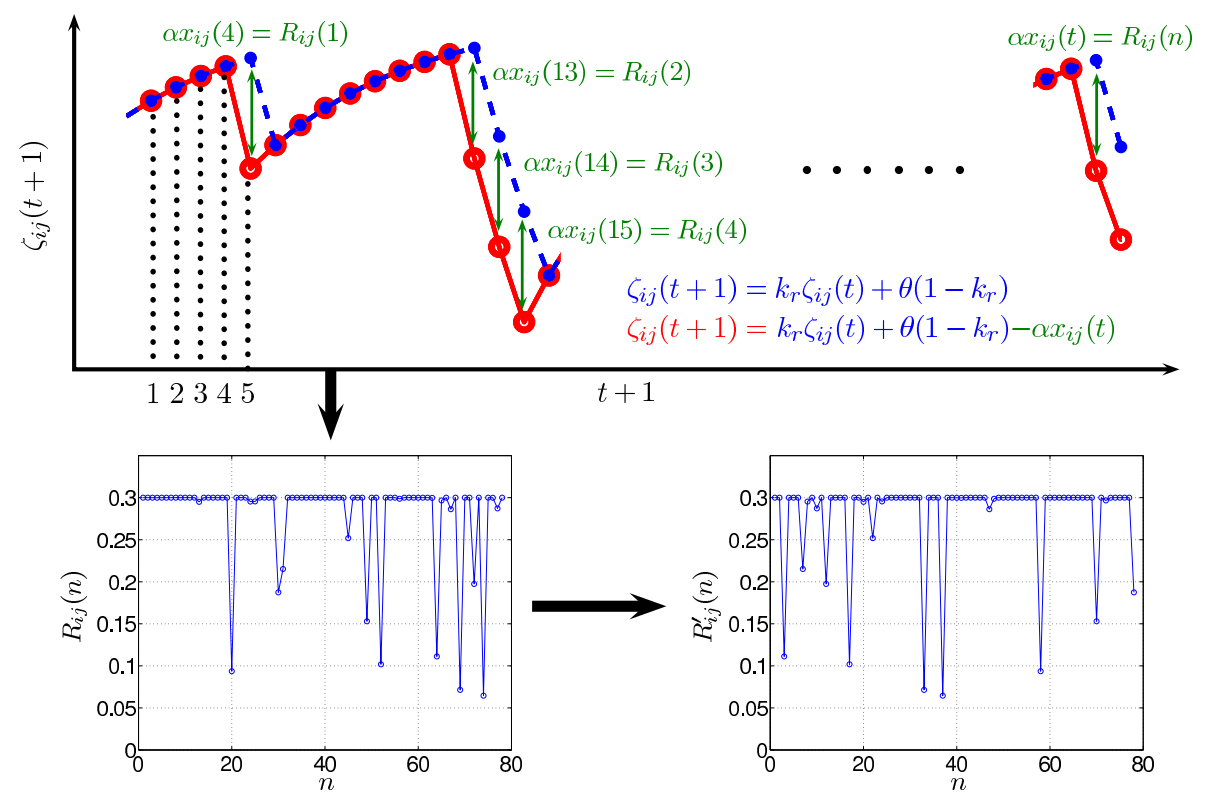

Fig. 9. Schematic representation of refractory effect time series of $R_{i j}(n)$. The time series of $R_{i j}(n)$ is the $n$th strength of the refractory effect after firing in the $i$ th chaotic neuron. In the CMS, $\alpha x_{i j}(t)$ corresponds to the strength of the refractory effect after neuron firings. $R_{i j}^{\prime}(n)$ is a randomly shuffled time series of $R_{i j}(n)$.

First, we introduce the random shuffled time series of $R_{i j}(n)$, denoted by $R_{i j}^{\prime}(n)$ (Case A). The frequency histogram of $R_{i j}^{\prime}(n)$ is exactly the same as that of the original $R_{i j}(n)$. As a result, we can obtain almost the same performance as that in the original case (Fig. 4 and 11(A)). This result indicates that it is important to adjust the frequency distribution of the strength of the refractory effect after neuron firing.

Next, we design a new surrogate time series data (Case B). The new time series data is only different from the time series of Case $\mathrm{A}$ in the frequency histogram, because the time series is constructed by uniform random numbers whose dynamic range is adjusted to be equal to $R_{i j}(n)$ for each neuron. Hence, if we cannot find motifs in Case B, the frequency histogram can be considered as an important factor in finding motifs efficiently. However, the success rate of Case B also becomes almost the same as the CMS (Fig. 11(B)). The result of Case B indicates that the increase in the strength of the 

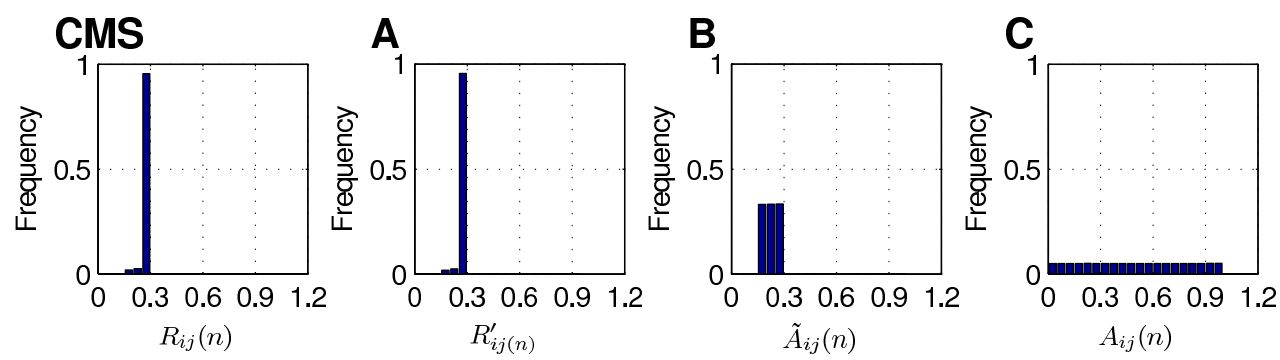

Fig. 10. Frequency histogram of the strength of the refractory effects after firing neurons. In CMS, frequency histogram of $R_{i j}(n)$, which is the value of the strength of the refractory effects after the $n$th firing of the $(i, j)$ th neuron in the CMS, is shown. In A, frequency histogram of $R_{i j}^{\prime}(n)$, which is a random shuffled time series of $R_{i j}(n)$, is shown. In $\mathrm{B}$, frequency histogram of $\tilde{R}_{i j}(n)$, which is a uniform random number whose dynamic range is adjusted to that of $R_{i j}(n)$ for all neurons, is shown. In $\mathrm{C}$, frequency histogram of $A_{i j}(n)$, which is the uniform random number between 0 and 1 ,is shown.

refractory effect after neuron firing is important but not in the frequency histogram for finding motifs efficiently.

Finally, we extract the motifs using the surrogate refractory effect of Case C. From Fig. 11(C), we can see that the success rates for Case $\mathrm{C}$ are lower than the success rates of the CMS. That is, motifs cannot be extracted by using Case C. This result indicates that it is important to adjust the strength of the refractory effect after neuron firing.

\section{Discussion}

In this paper, first, we investigated relationships between the positive Lyapunov exponents of the chaotic neuron and solving performance of the CMS. As a result, we found that the performance of the CMS does not depend on the positive values of Lyapunov exponents. Then, we examined the influence of the refractory effect in a chaotic search, which is probably a simple mechanism of memorizing the past searching history, in MEPs, which are combinatorial optimization problems. As a result, we found that motifs could not be extracted only if we applied surrogate refractory effects, for example a simple randomization or constrained randomization for the original refractory effect. These results indicate that the surrogate refractory effects cannot effectively control the neuron firings and the exploration in a solution space.

In the CMS, if a chaotic neuron fires, strength of the refractory effect becomes larger to inhibit a selection of same solution. On the other hand, the surrogate refractory effects do not exhibit such characteristic behavior because they are decided without considering the previous states of the neurons. Thus, it is considered that the strength of the refractory effects after the neuron firing is an important factor for the efficient exploration of the solution space. Then, we analyzed the strength of the refractory effects after the neuron firings. To analyze the effects, we used two types of random number and a simple randomization of the strength of the original refractory effects. As a result, the motifs can be found, if the dynamic range of the strength of refractory effects after neuron firing is the same as that of the CMS.

These results indicate that an important factor for finding near optimal solution of combinatorial optimization problem is not the chaotic dynamics but the inhibitions of selecting same solution. However, it needs to adjust the value of $k_{r}$, which controls decay speed of the refractory effect, because if the value of $k_{r}$ is slightly changed, both the CMS and the methods whose dynamic range of the strength of the refractory effect after neuron firing is the same as the CMS cannot find motifs.

The performance of the chaotic search has been investigated for MEP. However, the main concept of the study can be easily extended to different types of combinatorial optimization problems such as TSPs or QAPs. Hence, one of the important future works is to further analyze the high searching ability of chaotic neurodynamics for such problems.

In the CMS, it is not easy to evaluate whether the observed dynamics is chaotic by using Lyapunov exponents, because the dynamics of the chaotic neurons has the external input $\xi_{i j}(t)$. Then, in this 

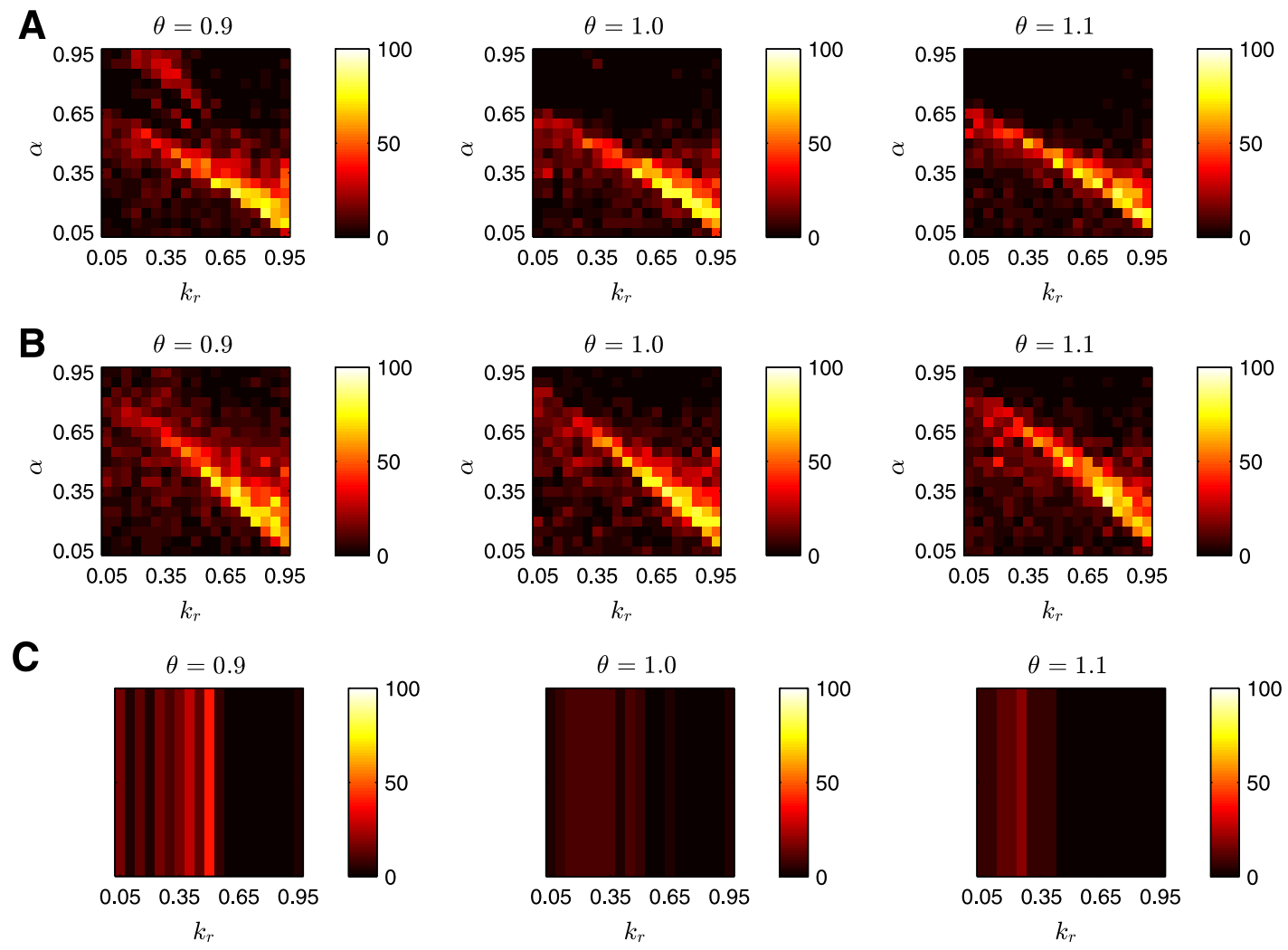

Fig. 11. Performance of the surrogate refractory effect after neuron firing. The average probabilities (\%) of finding motifs in 50 trials are indicated by the shading. To analyze the refractory effect in the chaotic neuron, the bifurcation parameters related to the refractory effect, $\alpha, k_{r}$, and $\theta$ are set to various values. The other parameters are fixed to $\beta=30.0$ and $\epsilon=0.01$. In (A), performance of the Case $\mathrm{A}$ is shown. In (B), performance of the Case $\mathrm{B}$ is shown. In $(\mathrm{C})$, performance of the Case $\mathrm{C}$ is shown.

paper, we only calculated the Lyapunov exponents of the chaotic neuron in the CMS when $\xi_{i j}(t)=0$, but we cannot find relation between the solving performance and positive Lyapunov exponents. Although it is not clear that the dynamics of chaotic neurons in the proposed method is chaotic, to deeper understanding of why the chaotic dynamics show high performance for combinatorial optimization, it is an important future issue to evaluate the solving performance relating to the Lyapunov exponents of the chaotic neurons with the external inputs. Then, another important future work is how to decide appropriate combination of values of parameters. To solve these issue, it is essential to analyze bifurcation structures of the chaotic neurodynamics.

\section{Acknowledgments}

The authors with to thank K. Aihara, Y. Horio, M. Adachi, N. Ichinose, and M. Hasegawa for their valuable comments and discussions. The research of T.M. is partially supported by a Grant-in-Aid from the JSPS Fellows (No.20.6863). The research of T.I. is partially supported by Grant-in-Aid for Scientific Research (B) (No.20300085) from the JSPS and research grant from the Mazda Foundation.

\section{References}

[1] M. Hasegawa, T. Ikeguchi, and K. Aihara, "Solving large scale traveling salesman problems by chaotic neurodynamics," Neural Networks, vol. 15, pp. 271-283, 2002.

[2] M. Hasegawa, T. Ikeguchi, and K. Aihara, "Combination of chaotic neurodynamics with the 2opt algorithm to solve traveling salesman problems," Physical Review Letters, vol. 79, pp. 2344$2347,1997$.

[3] M. Hasegawa, T. Ikeguchi, K. Aihara, and K. Itoh, "A novel chaotic search for quadratic assignment problems," European Journal of Operational Research, vol. 139, pp. 543-556, 2002. 
[4] T. Hoshino, T. Kimura, and T. Ikeguchi, "Two simple local searches controlled by chaotic dynamics for vehicle routing problems with time window," Proc. Metaheuristics Int. Conf. $2007,2007$.

[5] T. Hoshino, T. Kimura, and T. Ikeguchi, "A metaheuristic algorithm for solving vehicle routing problems with soft time windows by chaotic neurodynamics," IEICE Fundamentals, J90-A, no. 5, pp. 431-441, 2007 (in Japanese).

[6] T. Matsuura, T. Ikeguchi, and Y. Horio, "A tabu search and chaotic search for extracting motifs from DNA sequences," Proc. Metaheuristics Int. Conf. 2005, pp. 677-682, 2005.

[7] T. Matsuura and T. Ikeguchi, "Chaotic motif sampler: detecting motifs from biological sequences by using chaotic neurodynamics," Nonlinear Theory and Its Applications, vol. 1, no. 1, pp. 207-220, 2010.

[8] K. Aihara, T. Takebe, and M. Toyoda, "Chaotic neural networks," Physics Letters A, vol. 144, pp. 333-340, 1990.

[9] Glover F, "Tabu search I," ORSA Journal on Computing, vol. 1, pp. 190-206, 1989.

[10] Glover F, "Tabu search II," ORSA Journal on Computing, vol. 2, pp. 4-32, 1989.

[11] Y. Horio, T. Ikeguchi, and K. Aihara, "A mixed analog/digital chaotic neuro-computer system for quadratic assignment problems," Neural Networks, vol. 18, pp. 505-513, 2005.

[12] Y. Horio and K. Aihara, "Analog computation through high-dimensional physical chaotic neurodynamics," Physica D, vol. 237, pp. 1215-1225, 2008.

[13] H. Kantz and T. Schrieber, Nonlinear Time Series Analysis, Cambridge University Press, 2003.

[14] T. Matsuura and T. Ikeguchi, "Refractory effects of chaotic neurodynamics for finding motifs from DNA sequences," Lecture Note in Computer Science, vol. 4224, pp. 1103-1110, 2006.

[15] D. Hernandez, R. Gras, and R. Appel, "Neighborhood functions and hill-claiming strategies dedicated to the generalized ungapped local multiple alignment," European Journal of Operational Research, vol. 185, pp. 1276-1284, 2005.

[16] T. Akutsu, H. Arimura, and S. Shimozono, "Hardness results on local multiple alignment of biological sequences," IPSJ Digital Courier, vol. 3, pp. 174-182, 2007.

[17] C.E. Lawrence, S. Altschul, M. Boguski, J. Liu, and A. Neuwald, "Detecting subtle sequence signals: a gibbs sampling strategy for multiple alignment," Science, vol. 262, pp. 208-214, 1993.

[18] GenomeNet: http://www.genome.jp/ 